\title{
Criminologie
}

\section{Gérer les risques dans l'entreprise vulnérable. Une comparaison franco-québécoise}

\section{Frédéric Ocqueteau et Benoît Dupont}

Volume 46, numéro 2, automne 2013

Nouveaux regards sur les métiers de la sécurité

URI : https://id.erudit.org/iderudit/1020992ar

DOI : https://doi.org/10.7202/1020992ar

Aller au sommaire du numéro

\section{Éditeur(s)}

Les Presses de l’Université de Montréal

ISSN

0316-0041 (imprimé)

1492-1367 (numérique)

Découvrir la revue

Citer cet article

Ocqueteau, F. \& Dupont, B. (2013). Gérer les risques dans l'entreprise vulnérable. Une comparaison franco-québécoise. Criminologie, 46(2), 171-193. https://doi.org/10.7202/1020992ar
Résumé de l'article

Cet article est un essai de comparaison des résultats de deux enquêtes initialement conduites sans concertation à Paris et à Montréal sur un objet identique : la gestion de la sécurité-sûreté en entreprise à partir d'entretiens auprès de risk managers confrontés à des vulnérabilités, risques et menaces très diversifiés. L'approche française interroge plutôt compétences et rôles de ces agents d'encadrement à partir des cursus professionnels (privés, policiers, militaires) tandis que l'approche québécoise interroge plutôt la maîtrise et les effets de leurs réseaux relationnels. Le comparatif s'établit à partir des illustrations de deux régimes de sécurité : dans les structures hospitalières confrontées à des violences de plus en plus menaçantes et dans les aéroports vus comme des infrastructures critiques d'importance vitale. Une meilleure connaissance de l'identité de ces professionnels dédiés permet une approche plus juste de la pluralité des modalités du policing dans nos sociétés, une clé de lecture plus heureuse que celle du paradigme de la privatisation de la sécurité, dont la valeur heuristique est devenue moins crédible. 


\title{
Gérer les risques dans l'entreprise vulnérable. \\ Une comparaison franco-québécoise
}

\author{
Frédéric Ocqueteau ${ }^{1}$ \\ Directeur de recherche \\ Centre national de la recherche scientifique (CNRS) \\ Centre de recherches sociologiques sur le droit et les institutions pénales (CESDIP) \\ Université de Versailles-Saint-Quentin-en-Yvelines \\ ocqueteau@cesdip.fr \\ Benoît Dupont \\ Directeur \\ Centre international de criminologie comparée (CICC) \\ Université de Montréal \\ benoit.dupont@umontreal.ca
}

RÉSUMÉ - Cet article est un essai de comparaison des résultats de deux enquêtes initialement conduites sans concertation à Paris et à Montréal sur un objet identique: la gestion de la sécurité-sûreté en entreprise à partir d'entretiens auprès de risk managers confrontés à des vulnérabilités, risques et menaces très diversifiés. L'approche française interroge plutôt compétences et rôles de ces agents d'encadrement à partir des cursus professionnels (privés, policiers, militaires) tandis que l'approche québécoise interroge plutôt la maîtrise et les effets de leurs réseaux relationnels. Le comparatif s'établit à partir des illustrations de deux régimes de sécurité: dans les structures hospitalières confrontées à des violences de plus en plus menaçantes et dans les aéroports vus comme des infrastructures critiques d'importance vitale. Une meilleure connaissance de l'identité de ces professionnels dédiés permet une approche plus juste de la pluralité des modalités du policing dans nos sociétés, une clé de lecture plus heureuse que celle du paradigme de la privatisation de la sécurité, dont la valeur heuristique est devenue moins crédible.

MOTS-CLÉS • Comparaison France-Québec, régimes de sécurité, risk managers, sécurité hospitalière, sûreté aéroportuaire.

1. Frédéric Ocqueteau, Centre de recherches sociologiques sur le droit et les institutions pénales, Immeuble Edison, 43, boulevard Vauban, 78280 Guyancourt, FRANCE 


\section{Introduction}

Au fur et à mesure que s'accroissent la connaissance du policing dans les sociétés du risque (Beck, 2000) et les modalités concrètes de gouvernance de la sécurité (Dupont, 2007), les efforts naguère consentis pour démarquer les logiques de la sécurité publique de celles de la sécurité privée perdent progressivement de leur valeur heuristique. En effet, le classique distinguo entre une sécurité publique orientée vers la promotion de l'intérêt général sans redevabilité particulière à l'égard des citoyens, et une sécurité privée orientée vers la production d'un ordre instrumental redevable au client qui la rétribue, est devenu un trop facile artifice de lecture au regard de la complexité des situations rencontrées. Il est certain que l'impérieux besoin de théoriser la coexistence de l'ordre produit par l'intérêt général et l'intérêt commercial a été dicté par une anxiété de la criminologie radicale frappée par les développements d'une privatisation de la sécurité publique, dans une séquence historique dominée par les assauts du credo néolibéral et la progressive sortie de l'État-providence (Boltanski \& Chiapello, 1999). Si l'inventivité conceptuelle fut particulièrement féconde pour tirer des enseignements pratiques au sujet des nouvelles modalités de ce policing, il apparaît aujourd'hui que ces criminologies se sont trop rapidement embarquées dans des théorisations spéculatives assises sur de rares cas concrets, ne valorisant qu'oppositions et complémentarités, ratant une bonne part de leurs cibles. Pensons par exemple à la théorie du junior partnership (Kakalik \& Wildhorn, 1977), à celle du reflet (Brodeur, 1988), ou encore à celle des $4 C$ liée aux instrumentations réciproques de la complémentarité, de la concurrence, du contournement ou du conflit (South, 1988). Or, contrairement aux leçons tirées d'un cas concret resté emblématique (l'ordre du parc de loisirs Disney à Orlando, Floride) (Shearing \& Stenning, 1987), dont découla une vulgate retentissante sur les nouveaux traits de l'ordre disciplinaire contemporain dans les propriétés privées de masse, bien des exemples de dispositifs de sécurisation ultérieurement étudiés dans d'autres espaces hybrides montrèrent rapidement les limites de ce paradigme. C'est qu'y furent beaucoup plus souvent soulignées les interactions en ajustements croissants parmi clients, donneurs d'ordres, agents dédiés sous contrat ou salariés internes, publics cibles, ressources technologiques et humaines mobilisées et espaces de contrôle dans des modèles pluralistes. 
C'étaient là autant de dimensions et d'acteurs aux intérêts divergents qui rendaient moins crédible l'hypothèse d'une tendance univoque à la privatisation de la sécurité publique. En revanche, sous le coup d'une mutation progressive d'un «État garant» entré dans une gouvernementalité liée à la défense de la biosécurité (Gros, 2012), se répandait corrélativement une autre vérité. À savoir que la sécuritéprotection pouvait s'appréhender comme un bien privé normal, une marchandise utilement banale dans un processus général de marchandisation des services, plutôt qu'une valeur universelle fictive censée s'imposer obligatoirement à tous les citoyens et toutes les organisations par les voies d'un État garant ayant vécu. Aujourd'hui, les criminologues mettent plutôt en avant la coexistence d'une pluralité de «régimes de sécurité», modélisation qui présente l'avantage de mieux rendre compte de l'ensemble des facettes coexistantes du policing dans un monde en mutation dont le destin n'est pas écrit d'avance (Jones \& Newburn, 2006; Walle et al., 2012). Organisations et collectifs hommes-machines sont objectivement vulnérabilisés par une multiplicité de risques extérieurs et intérieurs qui les assaillent, et la mission des États s'en trouve d'autant réaménagée. Les institutions régaliennes ne s'affaiblissent pas nécessairement, bien au contraire, mais elles ne peuvent plus être pensées comme l'ultima ratio de la remise en ordre du monde. D'autres agents de sécurisation, quoique moins légitimes, mais peut-être plus efficaces, en débordent le périmètre à des échelons infra et supra nationaux (Hassid, 2010). C'est pourquoi, l'accent est plutôt mis sur les procédures de coordination multiniveaux et, à défaut de pouvoir encore en informer assurément tous les savoir-faire, se concentre sur les logiques de différents secteurs plus ou moins interconnectés, par le biais d'une analyse des différents régimes de sécurité (Dupont, 2013) en présence. On définit un régime de sécurité comme le point de convergence des forces et des contraintes qui déterminent les conditions dans lesquelles une organisation produit de la sécurité, pour elle-même ou pour autrui. Il délimite ainsi les termes du mandat confié par l'organisation à ses employés et à ses fournisseurs afin qu'ils la protègent contre une vaste panoplie de risques, ainsi que les moyens jugés acceptables pour y parvenir. Il influence également la propension et les capacités de cette dernière à collaborer avec d'autres organisations, ou au contraire à entrer en compétition ou en conflit avec elles, dans la perspective des réseaux de sécurité (Dupont, 2004). 
Cet article se situe dans le fil de cette problématisation émergente. À travers l'exemple de deux régimes de sécurité agençant des compétences et des ressources distinctes pour conjurer des menaces provoquant des vulnérabilités variables dans leurs entreprises, nous montrons comment les réseaux et le capital social des risk managers ${ }^{2}$ en façonnent concrètement le pilotage ou la gouvernance. Une hypothèse parcourt la démonstration: sur un continuum de vulnérabilités (d'intensité faible, moyenne, forte), les organisations les plus exposées aux risques majeurs instrumentalisent les ressources maximales de l'État dans leurs stratégies de prévention. À l'inverse, elles s'en détournent faute de pouvoir les instrumentaliser quand elles estiment que la masse des risques routiniers qui les affecte permet de les combattre de manière autonome. Mais dans la plupart des régimes de sécurité (monde hospitalier, monde du transport, mondes festifs...), au centre du continuum, se laisse percevoir une combinatoire de risques diffus et de risques majeurs qui rendent plus malaisées les grilles de lecture de la gestion purement publique ou purement privée. Les organisations concernées mobilisent le clavier de toutes les ressources dont elles disposent avec des moyens finis, donc beaucoup plus calculés. Et, dans ces conditions-là, la position et le profil du risk manager, outre ses compétences personnelles, doivent se comprendre à partir des atouts de son propre capital social (le profil de sa carrière antérieure) et/ou de son réseau relationnel, paramètres décisifs pour la maitrise et la mise en œuvre du régime de sécurité dont il assure le pilotage.

De ces deux dimensions, laquelle influence le plus l'art de prévenir les risques? Pour y répondre, nous avons estimé utile de passer par un exercice méthodologique de comparaison empirique. Est-ce la trajectoire personnelle du risk manager migré dans le privé après avoir fait sa carrière dans l'administration publique qui détermine au mieux les profils présents? (hypothèse française). Ne serait-ce pas plutôt la nature des réseaux maitrisés par le risk manager, bien plus que son appartenance d'origine, qui les déterminerait? (hypothèse québécoise). À défaut de trancher définitivement la question, il nous semble que la plus-value de l'exercice proviendra surtout d'une meilleure compréhension des ressorts différen-

2. Nous justifions cette terminologie générique par défaut, pour ne pas inférer de différences substantielles entre policing des risques liés à des malveillances ou actes hostiles (vols, prédations, agressions) et des événements catastrophiques naturels ou humains (incendies, paniques...). Dans l'entreprise vulnérable, les risk managers (ou faisant office de «monsieur ou madame sécurité») ne sont pas tous labellisés de la sorte, loin de là. Ce sont néanmoins tous des personnels d'encadrement détenteurs de talents spécifiques, qu'ils soient plutôt opérationnels (ils font) et/ou plutôt conceptuels (ils font faire). 
ciés parmi les acteurs concernés de leur degré d'incorporation du sens de l'intérêt général et/ou du pragmatisme attendu des résultats de la mission.

Un premier développement évoque le protocole de la recherche comparée, les panels mis en place à partir des deux postulations et justifie le choix de deux régimes de sécurité très différents. Le deuxième examine ce que disent et y font les risk managers, par le biais d'une lecture comparative de leurs trajectoires professionnelles et du tissu de leurs réseaux de relations. Le troisième discute des mérites respectifs de la connaissance de la trajectoire antérieure de l'acteur titulaire et/ou de son réseau de relations sociales dans les régimes de sécurité analysés.

\section{Protocoles, panels d'enquête et comparabilité illustrative}

L'échantillon parisien est constitué d'entretiens réalisés d'avril à septembre 2010 auprès d'un panel de 25 risk managers des secteurs privé, parapublic et public, menés aux sièges sociaux de leurs entreprises (Paris et Île-de-France). Ils furent sélectionnés avec l'aide du Club des Directeurs de Sécurité des Entreprises (CDSE), commanditaire de l'étude (Ocqueteau, 2011a; Ocqueteau, 2011b), ce qui a permis l'ouverture à la centaine de ses adhérents. Un cinquième d'entre eux fut sélectionné de la façon suivante: 20 adhérents du CDSE (et 5 non-adhérents) selon une stratégie de diversification maximale de leurs trajectoires réparties selon trois types de «filières»: 10 agents militaires (5 issus des services centraux ou opérationnels du renseignement extérieur et 5 officiers et sous-officiers issus de la Gendarmerie nationale); 5 agents du ministère de l'Intérieur reconvertis en fin de carrière, ou détachés de la Police nationale et des services préfectoraux; 10 agents issus de la «filière privée», à la tête d'entreprises privées ou issus de la promotion interne (des self-made-men experts en surveillance, incendie et risques informatiques, ou des ingénieurs et techniciens en fin de carrière). Âge et expériences antérieures (longues, courtes) dans le poste furent pris en compte, au sein d'entreprises elles-mêmes sélectionnées selon leur taille et leur degré d'exposition aux risques (entreprises à fort développement à l'international, «sensibles» ou non; entreprises au marché intérieur important, «sensibles» ou non; entreprises publiques à caractère industriel et commercial, administratif et culturel, et divers établissements recevant du public). Pour les besoins de la démonstration empirique, nous nous référons aux témoignages de sept acteurs du panel, dont trois 
occupent/ont occupé des fonctions de sécurité en structure hospitalière et quatre au sein d'aéroports de la région parisienne.

L'échantillon montréalais est constitué de 52 risk managers issus des secteurs privé, parapublic et public, avec qui des entrevues approfondies ont été menées entre janvier 2004 et mai 2005. Un échantillon initial a été constitué à l'aide de plusieurs annuaires spécialisés communiqués par des associations professionnelles comme ASIS International, BOMA, l'Association des directeurs de police du Québec et de l'Association canadienne des chefs de police. Une fois la première série d'entrevues réalisée, une liste complémentaire de répondants potentiels fut établie à partir des analyses préliminaires. Les 52 risk managers québécois de notre échantillon sont en grande majorité des hommes (77\%), dont l'expérience professionnelle dans le secteur de la sécurité est en moyenne de 20,2 ans. Un peu moins d'un tiers d'entre eux (32\%) ont entamé une seconde carrière après avoir pris leur retraite de la police, et une proportion équivalente $(37 \%)$ dispose d'un diplôme universitaire. Afin de diversifier profils, expériences et perspectives, nous avons localisé des risk managers opérant selon des paramètres économiques, réglementaires et culturels très différents. Ainsi, $40 \%$ travaillent dans des organismes parapublics, c'est-à-dire des services gouvernementaux ou des agences autonomes qui disposent de pouvoirs plus ou moins étendus d'enquête et d'application de la loi. La seconde catégorie de risk managers se trouve à la tête de services de sécurité internes dans des entreprises de taille très variées, comme des institutions financières, des commerces de détail, des entreprises de service ou encore des industries (31\%). Viennent ensuite les risk managers qui dirigent des entreprises prestataires de services et d'équipements de sécurité (la sécurité privée «à contrat»), (17\%). On trouve dans cette catégorie les filiales locales de grandes multinationales du gardiennage, des consultants très spécialisés dans la gestion des risques qui opèrent seuls, des sociétés de protection rapprochée, ou encore des installateurs de systèmes de vidéosurveillance et de contrôle d'accès. Le reste des répondants $(12 \%)$ provient des organisations policières et des associations professionnelles, ce qui nous a permis de bénéficier d'un regard extérieur sur les pratiques professionnelles et les stratégies institutionnelles des risk managers ${ }^{3}$. Pour les

3. Pour des raisons évidentes, les caractéristiques sociodémographiques de cette dernière catégorie de répondants «extérieurs» n'ont pas été incluses dans les chiffres présentés ci-dessus. 
besoins de la comparaison, treize entrevues ont été extraites de cet échantillon, trois acteurs relevant du milieu hospitalier et dix opérant dans l'environnement aéroportuaire.

La mise en commun de ces matériaux a rendu possible la comparaison franco-québécoise de deux régimes de sécurité, l'espace ayant manqué pour les mettre en regard avec un troisième, le régime de la grande distribution caractérisé par des risques et menaces d'intensité mineure ne mettant pas en cause la sécurité nationale. Il s'agit, d'une part, du régime de sécurité des établissements hospitaliers, certainement le plus délicat, puisqu'il demande de savoir y gérer en souplesse et aux moindres coûts au quotidien des populations en friction aux attentes et aux potentiels de dangerosité instables; d'autre part, du régime de la sûreté aéroportuaire, caractérisé au contraire par la nécessité d'assurer sans encombre les flux de passagers tout en sachant déjouer la menace suprême, l'attentat terroriste. L'État y garde la plupart des leviers, y compris s'agissant du screening des agents privés mobilisés dans la fouille des bagages et des passagers.

\section{Comparaisons des styles de risk management dans deux domaines sensibles}

Deux styles de management sont alternativement étudiés en France et au Québec: le premier concerne la sécurité dans un univers difficile mais pas encore considéré comme d'importance vitale (le monde hospitalier), le deuxième concerne la sûreté d'une infrastructure critique ou secteur dit d'importance vitale (les espaces aéroportuaires).

\section{Le management de la sécurité en structure hospitalière}

Depuis 1993, le souci de sécurité dans les structures hospitalières françaises, amené par l'expérience d'un ancien contrôleur de police reconverti associé à un travailleur social (Le Doussal \& Laures-Colonna, 1993; Le Doussal, 1995), n'a fait que s'amplifier à mesure que l'intolérance à certains seuils de malveillance ${ }^{4} s^{\prime} y$ serait fait sentir avec de plus en plus d'acuité. C'est ainsi que fut mis en place, une dizaine d'années

4. Ce concept flou fut précisément inventé par ce policier novateur, désireux d'établir un tableau de bord quantifié et standardisé de tous les incidents possibles survenant dans la structure hospitalière, non réductibles à des définitions strictement pénales ni à des incidents sans intentionnalité humaine, directe ou indirecte. 
plus tard, en 2005, un observatoire national des violences en milieu hospitalier centralisant des informations signalées par les établissements de santé, publics et privés. Sur $11 \%$ de la totalité des établissements $(\mathrm{n}=337$ sur 3140$)$ ayant signalé des faits dits de violence sur le territoire national, selon une nomenclature de gravité à quatre niveaux (Bauer \& Soullez, 2012, 874-879, singulièrement p. 879), le ministère de la Santé et des Sports en tire trois conclusions majeures: $86 \%$ des signalements de l'année 2011 émanent de centres hospitaliers, de centres hospitaliers spécialisés en psychiatrie et de centres hospitaliers universitaires. Un quart des atteintes aux biens et aux personnes ont été commises au sein des services de psychiatrie, les victimes d'auteurs agressifs étant majoritairement constituées par le personnel hospitalier; $95 \%$ des auteurs sont des patients pris en charge dans des services de psychiatrie et $67 \%$ dans des services de médecine. Le fait le plus notable et sans doute le plus problématique vient de ce que $15 \%$ des actes hostiles enregistrés sont imputables à des accompagnants ayant montré de l'agressivité verbale et physique envers les personnels administratifs et soignants.

Dans sa mission de pilotage de la sécurité à l'Assistance Publique - Hôpitaux de Paris (l'AP-HP regroupe 48 établissements de la région francilienne), l'actuel titulaire du poste n'est pas un policier ni un administrateur civil, mais, depuis 2008, un ancien officier de la Gendarmerie nationale. Il explique sans fard les raisons de son recrutement par la facilité relative avec laquelle il a su se glisser dans un poste devenu vacant et bien balisé, par son apprentissage récent à l'ouverture aux mondes qu'il ignorait. Se considérant comme doté d'une carrière classique faite de divers postes de terrain dans des structures territoriales de tailles différentes (arrondissements, départements), il insiste surtout sur la phase de son détachement au cabinet du préfet de police de Paris.

J'y ai beaucoup appris dans la connaissance d'un autre milieu, le relationnel et l'ouverture sur... (le monde), notamment, parce que la gendarmerie, c'est un peu fermé, c'est toujours un corps pas très ouvert, quoi...

Coopté par son prédécesseur à l'AP-HP, un administrateur civil ayant été lui-même en poste au cabinet du même PP, il estime, «après avoir appris à se positionner dans un ensemble qui n'est pas la gendarmerie et avoir chaussé les bottes de ses prédécesseurs», que la continuité des politiques de sécurité bien balisée y constitue un élément d'héritage 
important, sachant que la direction de l'AP-HP, tout comme celle des établissements connaissent en revanche un turnover important. L'une de ses missions prioritaires à la direction centrale consiste à sélectionner et à recruter les nouveaux chefs de sécurité dans l'ensemble des 48 établissements de l'AP-HP, et à leur enseigner les procédures efficaces à respecter en cas de conflictualité dégénérant entre personnel administratif et soignant, patients et familles.

Cette transformation de l'hôpital, d'un lieu de soins en un lieu où l'agressivité et la violence se manifestent avec une désarmante banalité, est un phénomène également très bien documenté en Amérique du Nord. Au Canada par exemple, en 2005, un sondage indique que $29 \%$ des infirmières avaient été physiquement agressées par un patient au cours des douze mois précédents, et que, parmi les hommes occupant ce type d'emploi, les taux de victimisation atteignaient $44 \%$ (Shields \& Wilkins, 2006: 37). Aux États-Unis, les incidents impliquant des armes à feu sont devenus courants dans les hôpitaux, y compris dans les services pédiatriques (Graham \& Shirm, 1995). Les risques d'agression découlent principalement des forts niveaux de stress associés pour les patients et leur entourage aux soins prodigués dans les services d'urgence, mais aussi, dans une moindre mesure, du mouvement de désinstitutionnalisation des services psychiatriques entrepris au cours des dernières décennies. Le risk manager d'un grand ensemble hospitalier regroupant 12000 employés explique ainsi comment ces transformations ont conduit ses équipes à développer une expertise dans le domaine de l'usage de la force:

Aussi, ils [les agents de sécurité sous sa responsabilité] sont intervenants sur des équipes de maîtrise physique de patients, des patients hors contrôle, les patients qui étaient psychiatrisés. Ce qui fait qu'on a beaucoup d'interventions, on doit adapter le service à ce changement-là... à l'hôpital XXX par exemple, nos interventions sur des patients augmentent de $20 \%$ par année, depuis quatre ans. Ce qui veut dire qu'on est actuellement dans une moyenne de 1000 interventions physiques par année... Les agents de sécurité font partie d'une brigade d'intervention, normalement, ils n'interviennent pas en bas de quatre personnes, ça veut dire que ça prend absolument deux préposés, deux agents et généralement, ils essaient d'intervenir à six.

La routinisation de l'usage de la force n'a toutefois pas totalement oblitéré les fonctions élargies d'assistance, qui restent omniprésentes et structurantes du «régime de sécurité» hospitalier. Mettre un hôpital en 
sécurité, selon cette risk manager, c'est aussi développer des systèmes de vigilance permettant de retrouver rapidement des patients fugueurs ne disposant pas de toutes leurs facultés mentales, afin de pouvoir assurer la continuité des soins qu'ils y reçoivent, ou encore privilégier le recrutement d'agents suffisamment patients et dotés d'un sens du jugement qui leur permette d'écouter et de rassurer les malades et leurs proches, plongés dans un environnement étranger et confrontés à des procédures qui les déstabilisent. Face à un personnel soignant surchargé, le dispositif de sécurité représente souvent l'une des rares sources d'interlocuteurs visibles et disponibles. La dimension budgétaire reflète également la tension quotidienne entre logique de sécurité et logique de soins. L'acquisition d'équipements de sécurité (surveillance, contrôle d'accès, boutons panique, etc.) n'est envisageable que si tous les besoins médicaux ont été pourvus:

Pour acheter de l'équipement neuf, on doit toujours faire des demandes pour avoir, par exemple, des systèmes d'enregistrement numérique (...). Et c'est souvent un choix entre un appareil médical ou un système d'enregistrement numérique, ils vont prendre l'appareil médical, ce qui est tout à fait normal. On vient toujours à bout de se débrouiller, sauf que le processus est beaucoup plus long.

Le «régime de sécurité» hospitalier reste par conséquent inféodé aux contingences médicales, même si, comme on l'a vu, l'évolution des relations patients-soignants a été marquée ces dernières années par une augmentation des actes de violence et par un recours de plus en plus fréquent à l'usage de la force. Sur le plan institutionnel, ce paradoxe se traduit par la nécessité pour les risk managers hospitaliers de négocier d'abord avec leur autorité de tutelle (le ministère de la Santé), plutôt qu'avec le ministère de la Sécurité publique ou les organisations policières, les paramètres opérationnels qui vont définir leurs pratiques.

Finalement, de quoi est fait l'apprentissage de cette nouvelle mission de sécurité interne à l'hôpital ? La réponse est à la fois simple et compliquée: en France, à l'échelon pyramidal de la plus grosse structure hospitalière étudiée, elle est entièrement dévolue à la mission d'expertise et de formation d'un officier de gendarmerie détaché, remarqué pour ses capacités d'ouverture, ayant appris, en même temps que les autres personnels hospitaliers, à gérer les conséquences de conflits latents souvent tus dans les enceintes de soins. De nombreux conflits sont désormais engendrés par le heurt frontal de différentes vulnérabilités, dont les victimes ou supposées telles revendiquent la priorité et le bon 
droit de la prise en charge sécurisante et sécuritaire, outre l'aide et les soins. Au Québec, cette mission est appréhendée sous un angle beaucoup plus managérial, même si les problèmes sont finalement très similaires. Une expérience policière préalable devient alors moins indispensable que la capacité à fonctionner dans un univers caractérisé par sa complexité et de nombreuses contradictions.

\section{Le management de la sûreté aéroportuaire}

En France, et notamment depuis le défi constitué par la montée des attentats terroristes et la suspicion induite par la possible présence de leurs auteurs dans les zones hautement vulnérables de transit aéroportuaire, la gestion pratique de la sûreté générale domine incontestablement celle de la sécurité publique. La privatisation de la fouille des bagages et de l'inspection des voyageurs dévolus à des agents contractuels inspectant leurs effets personnels ou se livrant à du profiling des comportements, ne pose plus, sauf exception, de soucis idéologiques majeurs (Gualino, 2012). En effet, ces missions s'exercent sous un contrôle permanent de divers corps d'inspection publics extrêmement attentifs au statut et au pouvoir des agents dédiés, ainsi qu'aux modalités de leur recrutement. Les tâches accomplies, généralement considérées comme ingrates et routinières, ne sont même plus revendiquées par les services publics, dans la mesure où l'on admet volontiers qu'il n'existerait pas d'avantage compétitif décisif entre un gardien de la paix et un agent privé, hormis le coût d'investissement dans ce type de mission, bien moins cher pour le second. Cet argument de la meilleure rentabilité étant admis, il semblerait que l'on ne puisse revenir en arrière, au point même d'admettre qu'à terme, les agents privés seraient devenus beaucoup plus efficaces dans leurs missions d'inspections et de fouilles que ne le seraient les anciens gardiens de la paix de la Police aux Frontières et autres agents des douanes qui s'acquittaient naguère de ce type de mission. La tentation américaine de créer des air marshals depuis le 09/11, une nouvelle administration de police dédiée à la sécurité à l'embarquement et en vol, autrement dit une «publicisation» de métiers auparavant privés, suscite en revanche l'ironie des fonctionnaires français. Ils louent plus volontiers l'expérience canadienne, laquelle aurait su inventer et mettre au point, de manière beaucoup plus pragmatique, un organisme administratif inédit au début des années 2000, l'ACSTA (Brodeur, 2006; Lyon, 2006). Il s'agit d'une société privée à capitaux 
publics sans but lucratif qui gère la passation des contrats et la surveillance opérationnelle des sous-traitants par le biais de la perception d'une taxe aéroportuaire acquittée par les passagers et rend compte au gouvernement fédéral. La tentation de «publicisation» formelle de telles missions n'est plus guère tenue que par certains syndicats de salariés français, et encore jusqu'à un certain point.

Telle n'est pas la pente qui se dessine en sûreté aéroportuaire, même si les points de vue des managers militaires et policiers ont pu largement diverger à ce sujet au milieu des années 2000. Soit, à titre d'illustrations, deux rhétoriques sensiblement différentes portées par deux personnages clés dont il importe ici de saisir la trajectoire personnelle pour comprendre la nature officielle et officieuse de leurs positions et pratiques effectives de pilotage sécuritaire. Pour cet ex-général de l'Armée de l'Air officiant à la direction de l'une des plus grosses entreprises de sûreté, le réalisme politique l'obligea à s'incliner en 2006 devant le refus fait à sa proposition de créer un secrétariat général à l'Air dont la tutelle eut dépendu du ministère de la Défense. Cette nouvelle direction aurait eu à ses yeux le mérite de particulariser la gestion du statut des quelque 10000 agents dédiés en France à ce métier, sachant que, comme l'affirme un témoin, la «sûreté aéroportuaire, qui n'est pas autre chose que de l'action antiterroriste, n'a rien à voir avec la sécurité générale accomplie par les vigiles contrôlant les sacs à main dans les grands magasins, vu que le terrorisme ne serait pas leur enjeu principal». Soucieux d'intégrer les représentations et points de vue de ses clients (ADP et compagnies aériennes), ce haut gradé de l'Armée de l'Air concède plus d'importance aux arguments financiers du jour, à la différence de ses homologues, des militaires de carrière opérationnels dont il épouse évidemment l'univers de valeurs, mais dont il lui importe néanmoins de se démarquer de la rhétorique réaliste:

À cette époque-là, quand vous disiez à un grand chef opérationnel: «On va estimer votre performance et on va voir comment vous dépensez l'argent qui vous est alloué», le gars vous répondait: «Moi, quand il y a la guerre, c'est là qu'on voit qui est le plus fort !» Je lui disais: «Ce n'est pas suffisant, la guerre, on ne va pas la faire tout de suite.» Donc, j'ai introduit le contrôle de gestion dans l'Armée de l'Air, mais déjà en 1998, avec la loi de programmation militaire 1995-2000, on commençait à rogner pas mal, donc on me demandait régulièrement, en tant que chef des finances de sortir 150 millions d'euros, boum... du soir au matin. Et donc, j'ai appris à compter à ce moment-là, j'étais un peu atypique (...). 
Ses anciennes compétences d'inspecteur général à la tête de l'Armée de l'Air ne lui ont pas fait oublier sa fonction préalable de $\mathrm{n}^{\circ} 2$ de l'étatmajor, de savoir, comme il l'explique dans l'entretien, compter les sous et compter les bonshommes. Elles lui ont surtout donné le goût de l'administration publique et expliqueraient largement les qualités recherchées dans son poste actuel. Elles ne lui ont pas fait oublier que, derrière le souci de la dépense publique, la nécessité d'améliorer l'image des agents privés de la sûreté aéroportuaire reste un impératif de tous les instants. Il rend hommage aux performances des agents de contrôle placés sous sa responsabilité, la ressource humaine restant déterminante par rapport aux ressources technologiques de la détection des cargaisons suspectes. Car, dans le grand écart permanent entre le régalien et le commercial, comme il le formule à trois reprises, la philosophie de la dissuasion prime à ses yeux sur tout le reste:

Les grands succès dans l'aéroportuaire, les grands terroristes arrêtés dans les vingt dernières années, l'ont d'abord été par les hommes, et pas par les machines. Sans doute parce que le terroriste s'adapte toujours à la machine, mais l'homme lui, ne peut pas s'adapter. Je vous donne trois exemples: Mlle M., une Irlandaise fiancée à un Palestinien, à qui il a dit: «Va en Israël, je te rejoins, et emmène-moi cette valise.» Elle a franchi tous les contrôles, c'était à Heathrow, et arrivée sur la passerelle, un agent de contrôle lui a dit: «Ben, madame, dans quel hôtel vous descendez?» Elle a dit: «Je sais plus quel hôtel, un hôtel cinq étoiles.» «Et comment vous comptez payer? Vous avez de l'argent?» «Non, je n'ai pas d'argent.» À partir de là, l'agent de sûreté lui a dit: «Venez me voir» et la valise avait un double fond, plein d'explosifs. Cette histoire est vraie (...). Donc, le savoir-faire de l'homme reste un paramètre très important, malgré les nouvelles technologies de détection.

Pour son homologue, le policier détaché à la Direction générale de l'Aviation civile (DGAC), les défis de la sûreté aéroportuaire se posent différemment. Placés sous la tutelle du ministère des Transports et du ministère de l'Intérieur, la formation et le savoir-faire des agents d'inspection restent un enjeu quasiment secondaire par rapport aux enjeux de la diplomatie interadministrative dont il faut savoir faire preuve; autrement dit, montrer sa bonne connaissance et maîtrise des rapports de force institutionnels entre des tutelles aux visions sécuritaires divergentes, puisque ciblées sur des finalités et des publics différents. Le témoin explique ainsi son entrée dans le poste par rapport à sa trajectoire professionnelle de commissaire de police: 
Les postes de sous-directeur de ce type-là sont publiés au Journal Officiel. Or, je me trouvais à la direction de la prospective et de la stratégie du ministère de l'Intérieur. Je lis le JO, je vois ce poste se libérer, donc je postule. Je rencontre mon prédécesseur, un administrateur civil et le directeur du transport aérien. J'en parle au DGPN et au DGAC et finalement, comme il y avait vraiment beaucoup de candidats, l'idée de mon recrutement qui s'est imposée fut la suivante: «On n'y avait pas pensé [à un commissaire], mais c'est pas idiot»... parce qu'en définitive, la sûreté aérienne est sous quatre tutelles. Le ministre des Transports qui est l'autorité compétente sur la réglementation européenne est donc leader, mais il doit composer avec le très gros ministère de l'Intérieur, composante police et gendarmerie, avec la Douane, avec le ministère de la Défense (...). Donc, on a une gouvernance de la sûreté compliquée, un peu éclatée. Et le fait de mettre un policier là, en 2008, avant c'était un administrateur civil, ce fut un pari de dire: «On va essayer de faire un rapprochement avec le ministère de l'Intérieur, ça permettra un peu de croiser les cultures, de faciliter les contacts.»

Son enjeu n'est pas tant d'améliorer les compétences, formations, acquis sociaux et pouvoirs liés au double agrément des agents affectés aux contrôles des bagages à main, de cabine, de soute et de soute accompagnée, dans la mesure où ces agents de première ligne seraient déjà les plus contrôlés de tous: la France, soumise comme ses homologues de l'Union aux législations européennes en matière de sûreté aérienne, aurait en effet créé un corps d'agents privés soumis aux réglementations les plus contraignantes qui soient. Les intérêts de la tutelle directe de la DGAC étant liés avant tout aux impératifs de satisfaction des clients, la contrainte d'investir encore dans la formation de ces agents afin qu'ils deviennent plus polyvalents, comme voudrait y obliger le ministère de l'Intérieur, fait l'objet de très fortes réticences au motif que la formation et les acquis sociaux des agents recrutés en sûreté aéroportuaire seraient, à ses yeux, à l'étiage maximal des possibilités légales. La justification ne manque pas d'intérêt:

Si ça continue ainsi, nos sociétés de sûreté aéroportuaire exerçant en France vont être plombées par rapport aux sociétés exerçant dans d'autres pays de l'Europe, parce que les coûts ne vont pas être les mêmes. Au lieu de simplifier comme en Belgique, qui applique directement le code européen, on développe en France de la technocratie administrative supplémentaire.

Au total, les nuances de positionnements par la différence des itinéraires et des intérêts défendus entre le militaire et le policier en sûreté aéro- 
portuaire pourraient paraître assez dérisoires. Il est vrai que pour les passagers confrontés aux contrôles et aux fouilles, le ressenti des contraintes de sûreté fait l'objet d'incessantes interrogations sur l'image de la sécurité, comme il en va dans le transport terrestre, et ce sont donc les questions de fluidité des flux qui priment et sont le plus valorisées chez les deux interlocuteurs. Dans la gestion interne de la sûreté implémentée au quotidien, les conflits de normes concurrentielles constituent néanmoins un enjeu sous-estimé par l'un, surestimé par l'autre. L'amélioration de la condition salariale des agents de première ligne fait l'objet d'âpres discussions feutrées montrant la profondeur de la divergence des intérêts défendus. Quand il s'agit de la gestion pratique, l'enjeu de la qualité et de la revalorisation de l'image générale des agents le dispute aux considérations de coûts des vacations horaires qui tendent à les occulter. Ces enjeux-là n'ont que très peu de retentissement médiatique problématique alors qu'ils sont majeurs en interne. Vu de l'extérieur, prédomine une indécente focalisation critique à l'égard d'agents localement recrutés parmi des minorités visibles, laquelle génère au contraire des soupçons d'accointances idéologiques avec des intégristes musulmans. Or, cela est d'autant moins problématique en interne que la direction des aéroports a directement accès à tous les renseignements souhaitables de cet ordre et n'a alors nul besoin de recourir à de la négociation pour savoir à quoi s'en tenir à leur sujet et pour exercer la brutalité de son pouvoir légal de licenciement à la moindre suspicion. Comme le résume un juriste spécialisé pour qualifier la menace islamiste radicale supposément favorisée par la présence de certains agents d'inspection privée: «En cas d'urgence, [leur] habilitation peut être immédiatement suspendue pour une durée maximale de deux mois, puis retirée, ce qui fut fait à diverses reprises vis-à-vis d'agents suspectés d'avoir des sympathies philosophiques pouvant inquiéter» (Belhache, 2012: 369).

Dans le cas québécois (et plus largement canadien, dans la mesure où le cadre réglementaire applicable aux zones aéroportuaires est de juridiction fédérale), et par contraste avec le «régime de sécurité» du monde hospitalier, une expérience antérieure dans une institution policière ou militaire semble hautement souhaitable pour accéder aux postes de gestion dans le monde de la sécurité aérienne. Alors que dans notre échantillon "généraliste», les policiers reconvertis ne représentaient que $32 \%$ des risk managers interviewés, cette trajectoire professionnelle semble nettement plus favorisée dans le domaine de la sécurité 
aérienne, puisqu'elle concerne $67 \%$ des répondants. Ces derniers opèrent dans un environnement où on observe une forte concentration géographique d'organisations publiques et privées de sécurité, ainsi qu'un enchevêtrement des liens de collaboration les unissant les unes aux autres. Pour l'Aéroport de Montréal, ce sont ainsi 30 acteurs de sécurité aux mandats et aux moyens extrêmement variés qui ont été identifiés, alors que sur le site de Toronto, un comité d'enquête parlementaire a identifié 56 organisations intervenant à un titre ou à un autre dans le dispositif de sécurité (Comité sénatorial permanent de la Sécurité nationale et la défense, 2003 : 137).

C’est bien entendu l'hégémonie exercée sur ce «régime de sécurité» par les risques liés à la sécurité nationale qui explique cette surreprésentation d'anciens policiers et militaires. Quatre grandes catégories de risques focalisent l'attention des intervenants: les risques liés au terrorisme font évidemment l'objet d'une prise en compte accrue depuis le 11 septembre. Les risques épidémiologiques font également l'objet d'une attention accrue depuis les alertes associées au SRAS (syndrome respiratoire aigu sévère) en 2002-2003, et à la grippe aviaire en 2009. De manière plus routinière, les risk managers sont également confrontés aux tentatives répétées d'infiltration des organisations criminelles organisées afin de faciliter, par des complicités internes, divers trafics. Enfin, certaines formes de petite délinquance, comme le vol à la tire, le vol de bagages ou le vol de véhicules de luxe, trouvent dans l'univers aéroportuaire un terreau particulièrement fertile. Même si l'impératif de rentabilité reste déterminant, ce qui implique par exemple que les interruptions aux flux de passagers fassent l'objet d'objections de la part des opérateurs privés qui cherchent à en réduire la durée au strict minimum, la sévérité des risques encourus (particulièrement sur les questions de terrorisme et de sanitaire) et l'emprise des autorités régulatrices font en sorte que les risk managers vont privilégier le travail en amont de la chaîne du risque, où ils disposent d'une plus large autonomie.

Ce travail en amont implique l'accès au renseignement, la maîtrise de méthodes d'analyse qui sauront le traduire en décisions opérationnelles, mais aussi l'établissement et l'alimentation de canaux d'échange de l'information qui vont renforcer la position du risk manager vis-à-vis des acteurs gouvernementaux. À titre d'exemple, il n'est pas anodin que le gestionnaire de sécurité de l'opérateur aéroportuaire ainsi que ses deux adjoints soient issus de la force de police provinciale, alors même que seules la police fédérale et la police municipale disposent d'une 
présence permanente sur le site. La logique implicite de l'opérateur aéroportuaire est de s'affranchir ainsi d'une dépendance exclusive à l'égard des deux organisations policières dominantes, tout en maintenant vis-à-vis des autres acteurs concernés une «neutralité » bienveillante propice au partage du renseignement. $S$ 'ils sont nombreux, ces échanges restent soumis à de fortes contraintes légales qui en restreignent la fluidité. Certains répondants ont ainsi décrit le ballet qui règne lors des réunions de coordination, au rythme des thèmes abordés: les risk managers doivent ainsi quitter la salle lorsque des dossiers portant sur des enquêtes criminelles sont abordés, seuls les agents de la paix assermentés pouvant accéder à certaines données nominatives tirées des fichiers policiers. Plus tard, lorsque des renseignements portant sur la sécurité nationale sont évoqués, certains des policiers présents qui ne disposent pas des habilitations de sécurité suffisantes doivent à leur tour se retirer temporairement, alors que les risk managers disposant des autorisations requises du fait de la nature sensible de leurs activités se joignent à la discussion. Ces allées et venues matérialisent les barrières réglementaires, mais aussi les privilèges et la hiérarchie informelle qui formatent les relations entre des gestionnaires aux intérêts et aux responsabilités parfois incohérents.

\section{Discussion comparative}

De l'analyse des situations respectives de ces deux régimes de sécurité, il ressort que la fonction émergente du risk manager est entièrement articulée à la recherche d'une meilleure maîtrise des risques par l'analyse et l'implémentation de dispositifs humains et technologiques dans des environnements incertains. Selon le degré d'ouverture et de fermeture aux publics des différentes organisations sécurisées auxquelles on a affaire, selon les besoins d'y hiérarchiser des menaces globalement considérées comme majeures ou mineures, l'influence des acteurs extérieurs sur le processus joue comme une variable déterminante. Les pouvoirs publics ont en théorie toujours la capacité d'imposer des dispositifs plus ou moins contraignants aux directions des entreprises (hétéronomie), et/ou de les laisser jouer leur propre partition en les invitant à mobiliser leurs ressources propres (autonomie). Sous l'impact de ces deux considérations macrosociologiques, les régimes de sécurité seront différemment affectés, ce qui reste vrai d'un côté de l'Atlantique comme de l'autre. 
La question de savoir comment, au sein des différents régimes, on met pratiquement en œuvre la protection des personnes et des biens et de l'information sensible, question à laquelle bien des sociocriminologues ont essayé de déduire un policing univoque pris dans les lames de fond d'une privatisation générale, constitue une entrée largement restée à l'état d'implicite. Ce n'est que lorsque l'on examine le statut de l'homme idoine convié à occuper une fonction de plus en plus stratégique que l'on entre véritablement au cœur de la compréhension des régimes de sécurité. Par-delà la façon dont elle est labellisée, on découvre bien alors d'autres utilités à la fonction.

L'homme-orchestre de la sécurité en entreprise (à la tête d'un service dédié) est la figure centrale d'une gestion systémique bien différente de celle que l'on rencontre en sécurité publique (Thoenig, 1994). Ses interlocuteurs majeurs restent avant tout les financiers de l'entreprise dont les yeux restent rivés sur le profit global. Tout son art va alors dépendre de sa capacité à surmonter les épreuves de sa propre profitabilité gestionnaire. Certes, il va être invité, tout comme les autres directions, à faire preuve d'adaptations dans l'invention permanente pour une meilleure maîtrise des coûts d'investissements dans la mise en œuvre de la protection des marchandises, des salariés et des publics. De ce point de vue, le risk manager issu d'une grande école de commerce peut être considéré comme un meilleur gestionnaire dans tel contexte plutôt que dans tel autre. S'il a les mains libres pour budgéter la politique de sécurité qu'il justifie par un savoir propre sur des menaces dont d'autres départements seraient dépourvus, il n'a pas toujours les mains aussi libres dans d'autres configurations. Du côté français, l'importance de la filière (privée/publique) d'où provient le titulaire du poste constitue un élément plus discriminant qu'il ne le serait au Québec.

Personne n'est assez naif pour imaginer qu'un homme seul, voire le dirigeant d'une petite équipe multicompétences, serait capable de mâ̂triser l'ensemble des vulnérabilités internes et externes d'une entreprise privée moyenne, a fortiori d'une organisation à dimension internationale. Plus la fonction en revanche touche à des enjeux stratégiques relevant du secret, plus la compétence attendue du risk manager devient conceptuelle, plus la délégation des tâches routinières est externalisée sous conditions, et plus la loyauté de l'agent devient un motif de poids dans son recrutement. Sa capacité d'obtenir du renseignement stratégique pour le compte de son entreprise et de savoir le négocier au mieux, au bon moment, est sans aucun doute l'atout déterminant du risk manager 
privé (ou privatisé) de demain, avec toutes les dérives que de tels processus peuvent engendrer (Fansten, 2012). Dans les régimes hybrides et publics français en revanche, on conçoit mieux que la figure du risk manager demeure périphérique ou subordonnée à d'autres départements stratégiques. Ce qui préoccupe et conditionne le plus le choix des candidats dans les entreprises publiques ou parapubliques, c'est l'organisation de la migration et de l'apport personnel du haut fonctionnaire militaire ou policier dans le poste. Ce sont toujours les états de service et compétences reconnues dans leurs carrières antérieures qui dominent les processus électifs de ces personnels détachés ou reconvertis. On rencontre d'ailleurs généralement plus de policiers en détachements temporaires dans les structures parapubliques, voire privées ${ }^{5}$, que de militaires reconvertis en fin de carrière dans toutes les sortes d'entreprises.

Deux paramètres majeurs expliquent de tels usages en France. S'agissant des recrutements policiers, c'est plus leur sens de la diplomatie attestée par des expériences précédentes qui est recherché. Or, il semble qu'ils aient encore beaucoup plus de mal à forcer la porte des entreprises que ne le font les militaires, ayant généralement plus à y perdre qu'à y gagner. Tout autre est l'attitude des militaires ou officiers de gendarmerie. Outre de plus grandes facilités de cumul avec leur retraite arrivée relativement jeune, ce sont plutôt les vertus liées à leur habitus d'obéissance aux directions d'entreprise qui sont valorisées, quand l'ordre interne paraît s'y décomposer. Quoi qu'il en soit, on escompte toujours de ces deux types de fonctionnaires mobilisés des retours sur investissement en termes de facilitation d'accès aux renseignements stratégiques de l'État et de mobilisation des acteurs publics en cas de crise majeure, bien plus que de s'attendre aux effets vertueux de leurs compétences gestionnaires, même si elles peuvent être indéniables.

L'environnement institutionnel dans lequel les organisations chargées de la sécurité publique interagissent avec les prestataires privés joue un rôle également déterminant dans la nature des propriétés associées aux divers régimes de sécurité. Au modèle binaire français, articulé autour d'un

5. Pour une exception significative, voir Querry \& Ocqueteau, 2011 : 41. R.-G. Querry, ex-directeur du Service de Coopération Technique Internationale de Police (SCTIP), fut un grand policier reconverti dans la sécurité du Groupe Accor (hôtellerie). Il avoue sans ambages: «Je pense que l'un des éléments déterminants ayant justifié mon recrutement a été le réseau que je possède au plan national et international.» 
duopole police-gendarmerie, s'oppose une configuration décentralisée, dont le Québec représente un exemple typique. La taille réduite des organisations policières, ainsi que la nature locale de leur mandat, les amène à développer de manière inévitable des partenariats avec leurs homologues locaux et nationaux, mais aussi avec une grande diversité d'acteurs communautaires et privés. Autrement dit, les moyens limités dont disposent les services de police québécois, par contraste avec les ressources beaucoup plus conséquentes de leurs cousins français, les disposeraient à fonctionner de manière moins insulaire et à nouer plus spontanément des liens destinés à faciliter leurs interventions dans les domaines les plus variés avec les secteurs parapublic et privé. Cela se traduit sur le terrain par l'émergence de réseaux de sécurité hybrides caractérisés par une forte densité et une interdépendance marquée (Dupont, 2006), ou encore par le décloisonnement des forums professionnels dans lesquels policiers et risk managers mettent leurs expériences en commun. À titre d'exemple, l'Association canadienne des chefs de police (l'équivalent du Syndicat des Commissaires de la Police Nationale français) accueille parmi ses membres des risk managers et a constitué un comité de liaison avec le secteur privé dont l'objectif explicite est de favoriser une relation durable entre les secteurs public et privé pour la collecte et le partage des informations sélectionnées. Si ces interactions répétées favorisent évidemment la reconversion des policiers désirant entreprendre une seconde carrière, elles permettent aussi aux risk managers issus du monde de l'entreprise de s'insérer plus aisément dans des réseaux de partage de l'information dont les modalités d'accès semblent moins exclusives au Québec qu'en France.

L'étude de ces deux régimes de sécurité ancrés dans deux configurations nationales fort différentes montre bien à quel point les variations observées d'un site à un autre peuvent difficilement être analysées à l'aide de cadres théoriques trop rigides. Les objectifs, les ressources et les logiques déployées par les acteurs non étatiques de la sécurité constituent un gisement de matériaux empiriques encore sous-exploité par les chercheurs, qui se sont peut-être trop rapidement satisfaits d'expliquer par la privatisation ou la marchandisation les transformations en train de se dérouler sous leurs yeux. À travers l'approche comparative, il nous a plutôt semblé utile de souligner les limites aux transferts conceptuels hasardeux trop fréquemment utilisés dans la littérature, par lesquels certains cas extrêmes de privatisation de la sécurité se trouvent érigés en nouvelles normes mondialisées signalant 
le déclin irrémédiable de l'État protecteur. Bien au contraire, les cultures nationales et locales, les paramètres historiques, économiques, organisationnels - et même personnels - propres à chaque régime font éclore une myriade d'arrangements qu'il convient d'examiner en se gardant de tout a priori. S'ils n'ont pas toujours l'élégance de se conformer aux récits dominants sur la société du risque (Ericson \& Haggerty, 1997; Beck, 2000), ces régimes pluriels ont au moins le mérite de nous rappeler que face aux vulnérabilités, le nuancier de la sécurité ne se limite pas qu'à deux couleurs.

\section{Références}

Bauer, A., \& Soullez, C. (2012). Les actes de violences en milieu hospitalier en 2011 recensés par l'ONVH. In A. Bauer et C. Soullez (eds.), La criminalité en France, Rapport 2012 de l'Observatoire national de la délinquance et des réponses pénales (874-879). Paris: CNRS-INHESJ.

Beck, U. (2000). La société du risque. Paris: Aubier.

Belhache, C. (2012). Guide pratique et commenté du droit et des métiers de la sécurité privée (6 éd.). Paris: Secudoc SNES Éditions.

Boltanski, L. \& Chiapello, E. (1999). Le nouvel esprit du capitalisme. Paris: Gallimard.

Brodeur, J.-P. (1988). Ordre public et ordre privé. Revue internationale de criminologie et de police technique, 4, 392-410.

Brodeur, J.-P. (2006). Le transport aérien et la gestion des risques. Introduction. Revue canadienne de criminologie et de justice pénale, 48(3), 333-344.

Comité sénatorial permanent de la sécurité nationale et de la défense. (2003). Le mythe de la sécurité dans les aéroports canadiens. Ottawa: Parlement du Canada.

Dupont, B. (2004). Security in the age of networks. Policing and Society, 14(1), 76-91.

Dupont, B. (2006). Delivering security through networks: Surveying the relational landscape of security managers in an urban setting. Crime, Law and Social Change, 45(3), 165-184.

Dupont, B. (2007). La gouvernance et la sécurité. In M. Cusson, B. Dupont et F. Lemieux (eds.), Traité de sécurité intérieure (67-80). Montréal: Cahiers du Québec.

Dupont, B. (2013). Private security regimes: Conceptualizing the forces that shape the private delivery of security. Document inédit.

Ericson, R., \& Haggerty K. (1997). Policing the risk society. Oxford: Oxford University Press.

Fansten, E. (2012). Les nouveaux barbouzes, enquête sur la privatisation de l'espionnage. Paris: Laffont. 
Graham, C., \& Shirm, S. (1995). Security in pediatric emergency departments. Pediatric Emergency Care, 11(4), 220-222.

Gros, F. (2012). Le Principe Sécurité. Paris: Gallimard.

Gualino, M. (2012). La place des opérateurs dans la gouvernance de la sûreté du transport aérien. Cabiers de la sécurité, 19, 74-80.

Hassid, O. (2010). Les dynamiques actuelles du marché de la sécurité en France. Champ Pénal/Penal Field, VII. Consulté le 30 mai 2013, http://champpenal. revues.org/7926.

Jones, T., \& Newburn, T. (2006). Plural policing, a comparative perspective. London: Routledge.

Kakalik, J. S., \& Wildhorn, S. (1977). The private police: Security and danger. New York: Crane Russak.

Le Doussal, R. (1995). À l'hôpital: anti-malveillance et technologies. Les cabiers de la sécurité intérieure, 21, 75-87.

Le Doussal, R., \& Laures-Colonna, P. (1993). La sécurité à l'hôpital. Paris: ESF éditions.

Lyon, D. (2006). Airport screening, surveillance and social sorting: Canadian responses to 9/11 context. Revue canadienne de criminologie et de justice pénale, 48(3), 397-412.

Ocqueteau, F. (2011a). Profils et trajectoires des directeurs sûreté. Résultats d'une enquête conduite auprès de 25 grandes entreprises. Sécurité et Stratégie, 5, 39-53.

Ocqueteau, F. (2011b). Chefs d'orchestre de la sûreté des entreprises à l'ère de la sécurité globale / Heads of corporate security in the era of global security. Champ Pénal/Penal Field, VIII. Consulté le 30 mai 2013, http:// champpenal.revues.org/8142

Querry, R.-G., \& Ocqueteau, F. (2011). Sécurité de l'entreprise et défense de la réputation de la marque. Entretien avec René-Georges Querry, Groupe Accor. Sécurité et Stratégie, 6, 39-48.

Shearing, C. D., \& Stenning, P. C. (1987). Say "Cheese! ": The Disney order that is not so Mickey Mouse. Private policing. Newbury: Sage.

Shields, M., \& Wilkins, K. (2006). Findings from the 2005 national survey of the work and bealth of nurses. Ottawa: Statistics Canada.

South, N. (1988). Policing for profit, the private security sector. London: Sage.

Thoenig, J.-C. (1994). La gestion systémique de la sécurité publique. Revue française de sociologie, $X X X V(3)$, 357-392.

Walle, G. V., Herrewegen, E., \& Zurawski, N. (2012). Crime, security and surveillance, effects for the surveillant and the surveilled. The Hague: Eleven international publishing.

ABSTRACT - This article attempts to compare the results of two research projects conducted independently in Paris and Montreal on the same topic: the management of corporate security-safety understood through the eyes of risk managers who face a diversified pool of vulnerabilities, risks and threats. The French component of this article investigates the competencies and roles of security managers, based on their 
professional experiences (exclusively private, former police and military officers), while the Quebec component highlights how these individuals design and exploit their relational networks. The comparison revolves around empirical data drawn from two security regimes: the more violence-prone hospital environment and the strategic critical infrastructures of global mobility that are international airports. A more detailed understanding of these risk managers' professional identity allows us to contribute to the plural policing approach, which in our opinion represents a more fertile interpretative framework than the privatization paradigm, whose heuristic value is losing its currency.

KEYWORDS - France - Quebec comparison, security regimes, risk managers, hospital safety, airport security.

RESUMEN - El presente articulo es un atisbo de comparación de los resultados de dos estudios llevados a cabo sin concertación en Paris y en Montreal sobre un objeto idéntico: la gestión de la seguridad en las empresas a partir de entrevistas a gerentes de riesgos (risk managers) confrontados a vulnerabilidades, riesgos y amenazas muy diversificadas. El enfoque francés interroga sobre todo las competencias y los roles de dichos agentes de riesgo a partir de cursos profesionales (privados, policíacos, militares), mientras que el enfoque quebequense interroga más bien el manejo y los efectos de sus redes de relaciones. La comparación se establece a partir de ilustraciones de los dos regímenes de seguridad: en las estructuras hospitalarias confrontadas a violencias cada vez más amenazantes y en los aeropuertos, vistos como infraestructuras criticas de una importancia capital. Un mejor conocimiento de la identidad de estos dedicados profesionales permite un enfoque mas acertado de la pluralidad de modalidades del policing en nuestras sociedades, una clave de lectura más apropiada que aquella del paradigma de la privatización de la seguridad, de la cual el valor heurístico ha devenido menos creíble.

PALABRAS CLAVE - comparación Francia-Québec, regímenes de seguridad, gerentes de riesgo (risk managers), seguridad hospitalaria, seguridad aeroportuaria. 